\title{
Influence of dimer buckling on dimer diffusion: A scanning tunneling microscopy study
}

\author{
Jeroen Huijben, Arie van Houselt, Harold J. W. Zandvliet, and Bene Poelsema \\ Solid State Physics Group, MESA + Institute for Nanotechnology, University of Twente, P.O. Box 217, \\ 7500 AE Enschede, The Netherlands \\ (Received 28 July 2005; revised manuscript received 10 November 2005; published 23 February 2006)
}

\begin{abstract}
The diffusion of $\mathrm{Ge}$ dimers along the substrate dimer rows of $\mathrm{Ge}(001)$ has been investigated with scanning tunneling microscopy. The jump frequency of on-top Ge dimers along symmetric dimer rows at room temperature is found to be eight times higher than the diffusion along asymmetric dimer rows $\left(0.36 \mathrm{~s}^{-1}\right.$ versus $\left.0.044 \mathrm{~s}^{-1}\right)$. We ascribe this difference to limitations associated with the rocking motion that a dimer has to perform while diffusing along asymmetric dimer rows.
\end{abstract}

DOI: 10.1103/PhysRevB.73.073311

PACS number(s): 68.35.Fx, 68.37.Ef, 68.35.Bs

\section{INTRODUCTION}

The diffusion of atoms on a solid surface is a fundamental problem in surface science that has attracted attention for several decades. Also, from a technological viewpoint, surface diffusion is a very important topic because it plays a key role in, e.g., crystal growth, etching, and the stability of adstructures. For instance a good understanding of surface diffusion may lead to a better control over growth conditions and the tailoring of these to obtain atomically sharp interfaces. The diffusion of $\mathrm{Si}(\mathrm{Ge})$ dimers on $\mathrm{Si}(001)$ and $\mathrm{Ge}(001)$ surfaces has been studied elaborately and these systems act as model systems. ${ }^{1-9}$ No doubt the driving force behind this is that $95 \%$ of the present microelectronic devices use a $\mathrm{Si}(001)$ substrate as a basis.

The Ge(001) surface is more or less a look alike of the $\mathrm{Si}(001)$ surface. ${ }^{10,11}$ Both surfaces dimerize and exhibit a double domain $(2 \times 1)$ reconstruction due to perpendicular alignment of dimer rows terminating each of the two fcc sublattices that constitute the diamond lattice. Actually, dimers are observed in two apparently different forms, called symmetric and asymmetric dimers. ${ }^{12}$ An asymmetric dimer has one atom that buckles out of the surface plane and one atom that buckles inwards. It is now generally accepted that dimers, which appear symmetric in scanning tunneling microscopy (STM) images are in fact rapidly switching between two equivalent asymmetric geometries, in which the role of the out- and inwards buckled atoms is reversed. ${ }^{13,14}$

The aim of this paper is to study the influence of dimer buckling of the substrate dimer rows on the diffusion of an ad-dimer over the substrate dimer rows. The diffusion of an ad-dimer along an asymmetric (buckled) dimer row turns out to be significantly slower than along a seemingly symmetric substrate dimer row. We propose that this difference in diffusion rate is due to the fact that the ad-dimer has to perform a wiggling motion when it diffuses along an asymmetric dimer row.

\section{EXPERIMENTAL}

The experiments were carried out in an ultrahigh vacuum (UHV) system equipped with a scanning tunneling microscope and a base pressure of $3 \times 10^{-11}$ Torr. After outgassing the $\mathrm{Ge}(001)$ samples at $700 \mathrm{~K}$ they were further cleaned by cycles of $\mathrm{Ar}^{+}$ion bombardment $\left(800 \mathrm{eV}, 2 \mu \mathrm{A} / \mathrm{cm}^{2}\right.$, angle of incidence $45^{\circ}$ for $30 \mathrm{~min}$ ) and annealing at $1100 \mathrm{~K}$ for a few minutes. During annealing the pressure did not rise above $3 \times 10^{-10}$ Torr. This procedure resulted in atomically clean $\mathrm{Ge}(001)$ surfaces exhibiting an ordered $(2 \times 1) / c(4$ $\times 2$ ) domain pattern with a low concentration of missing dimer defects. After equilibration to room temperature a very low concentration $(\ll 0.01 \%$ of a monolayer) of $\mathrm{Ge}$ addimers is found on the surface. Several of these ad-dimers are selected and imaged for time lapses as long as a day.

\section{RESULTS AND DISCUSSION}

The first real-space diffusion studies of $\mathrm{Si}$ dimers on Si(001) were reported by Dijkkamp, Van Loenen, and Elswijk. ${ }^{15}$ With a scanning tunneling microscope held at a stable temperature ranging from room temperature to $400 \mathrm{~K}$ they were able to observe "in real space" the preferential diffusion of Si dimers along the substrate rows. Several years later, Swartzentruber ${ }^{1}$ used tracking tunneling microscopy to measure this diffusion process with a much higher time resolution and found a diffusion barrier of $0.94 \pm 0.09 \mathrm{eV}$ and an attempt frequency of $10^{12.8 \pm 1.3} \mathrm{~Hz}$. The diffusion barrier of Ge dimers along the substrate rows of $\mathrm{Ge}(001)$ is significantly lower, namely $0.82 \pm 0.05 \mathrm{eV}$ (which translates into $\sim 1$ hop per 9 seconds). ${ }^{8,9}$ It should be reiterated that in the latter analysis no difference was made between diffusion along seemingly symmetric substrate dimer rows and asymmetric substrate dimer rows. The influence of a scanning tip on the diffusion of a Si dimer was shown to be very small in tracking tunneling microscopy. ${ }^{16}$ This method keeps the scanning tip in close proximity of the diffusing dimer whereas in the measurements described in this communication the tip was only close to the dimer for about $2 \%$ of the time, reducing its influence even further.

Figure 1 shows two images of the same Ge ad-dimer diffusing at room temperature along a substrate dimer row on $\mathrm{Ge}(001)$ in an area free of defects that could disturb its motion. We have collected successive images of the same area of the surface for more than $12 \mathrm{~h}$ while keeping the temperature stable at $298 \mathrm{~K}$. Examination of the total video reveals that the diffusion of the on-top Ge ad-dimer along a seem- 


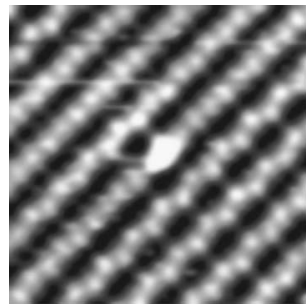

a)

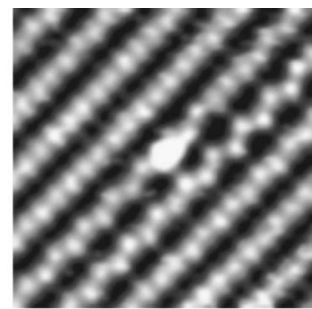

b)

FIG. 1. Scanning tunneling microscopy images of a Ge on-top ad-dimer diffusing along the substrate dimer rows of Ge(001). Symmetric substrate dimer row (a) asymmetric substrate dimer row (b). Sample bias $-1.6 \mathrm{~V}$, tunnel current $0.5 \mathrm{nA}$ on image size $5 \mathrm{~nm}$ $\times 5 \mathrm{~nm}$.

ingly symmetric substrate dimer row is much faster as compared to the diffusion along an asymmetric (buckled) substrate dimer row. Because of the former and the fact that the fractions of symmetric dimer rows and asymmetric dimer rows are about equal at room temperature, the dimer diffuses along an asymmetric dimer row for about $90 \%$ of the time. Therefore we do not have enough statistics to accurately study the mean-square displacement for the diffusion over seemingly symmetric rows on time scales longer than 1000 s. Figure 2 shows the mean-square displacement versus time. For diffusion of the dimer along asymmetric dimer rows the mean-square displacement is found to scale linearly with time, i.e., $\left\langle x^{2}(t)\right\rangle=a^{2} \nu t$, where $a$ is the surface lattice constant and $\nu$ the hopping frequency. The respective hopping frequencies, $\nu_{S}$ and $\nu_{A S^{\prime}}$, are thus exactly the slopes in Fig. 2. The hopping frequencies for diffusion along seemingly symmetric and asymmetric dimer rows are $0.36 \mathrm{~s}^{-1}$ and $0.044 \mathrm{~s}^{-1}$, respectively. In order to figure out whether the difference in hopping frequencies is due to a difference in attempt frequencies or diffusion barriers, temperature dependent experiments are required. A difference in attempt frequency between both processes would lead to a constant factor between both hopping frequencies independent of the actual temperature.

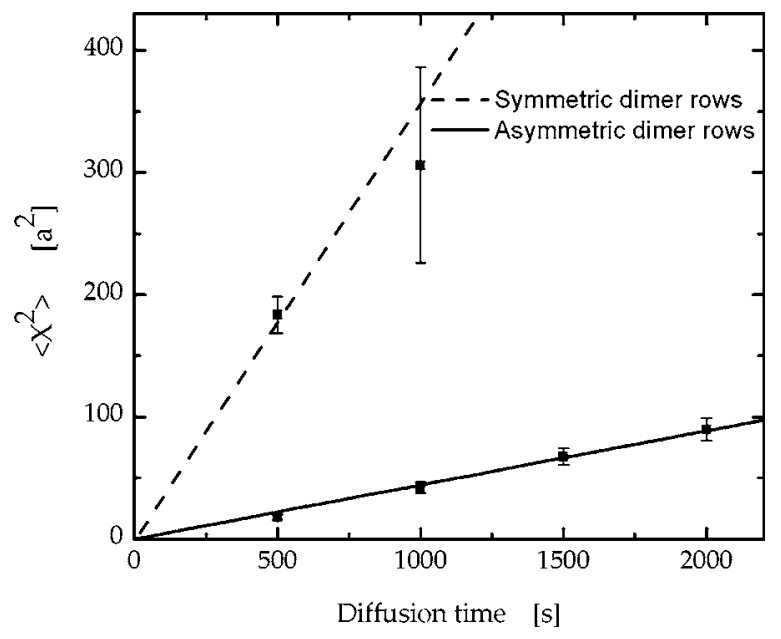

FIG. 2. Mean-square displacement of a diffusing Ge dimer along symmetric dimer rows (dashed line) and asymmetric dimer rows (solid line) versus time. ( $a$ is the surface lattice constant, $a$ $=4 \AA$.)

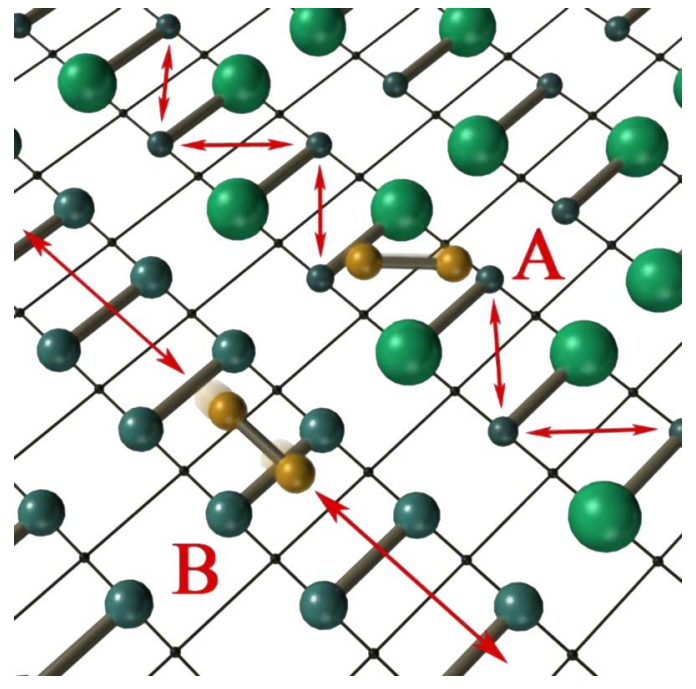

FIG. 3. (Color online) Schematic model of an on-top ad-dimer diffusing along an asymmetric substrate dimer row $(A)$ and along a symmetric substrate dimer row $(B)$. The symmetric appearing substrate dimers actually flip-flop very rapidly between the two buckled states.

A difference in diffusion barrier (attempt frequencies the same) would however lead to a decrease of $\nu_{S} / \nu_{A S}$ with increasing temperature (from 8 at $300 \mathrm{~K}$ to 6.6 at $330 \mathrm{~K}$ ). Experimental constraints do not allow us to perform these kinds of experiments because the fraction of symmetric appearing dimers rapidly grows at the expense of asymmetric dimers with increasing temperature. ${ }^{17}$ Already at $330 \mathrm{~K}$ the $\mathrm{Ge}(001)$ surface is fully comprised of symmetric appearing dimers. Of course this still allows one to measure $\nu_{S}$ as a function of the temperature and extract via an Arrhenius plot both the attempt frequency and diffusion barrier. But this analysis would not solve the aforementioned problem whether the difference in $\nu_{S}$ and $\nu_{A S}$ is in the attempt frequencies or diffusion barriers.

Figure 3 shows sketches of a Ge ad-dimer diffusing along a symmetric substrate dimer row $(A)$ and an asymmetric substrate dimer row $(B)$. It is immediately clear from Fig. 3 that the diffusing Ge ad-dimer can conserve its ideal $B$-type orientation (dimer bond of the $\mathrm{Ge}$ ad-dimer is perfectly aligned along the substrate dimer row direction) during the diffusion process along a symmetric row. In contrast, the Ge ad-dimer that diffuses along the buckled substrate dimer rows has to adapt its location alignment with respect to the underlying substrate dimer row when it hops to a neighboring site (adjacent dimers in a dimer row are always buckled in an asymmetric fashion). One should realize that the symmetric appearing substrate dimer rows actually consist out of dimers that continuously flip-flop between their two buckled configurations with a frequency that is much higher than can be measured with STM. Moreover, the dimers will most likely exhibit this flip-flop motion in a concerted manner. Therefore, it is likely that during this flip-flop motion the frequency factor and/or diffusion barrier to hop to an adjacent site will vary. 


\section{CONCLUSIONS}

The diffusion of on-top Ge ad-dimers along the substrate dimer rows of a $\mathrm{Ge}(001)$ surface is studied with scanning tunneling microscopy. We found a significant difference in jump rate for a dimer that diffuses along a symmetric appearing dimer substrate row as compared to an asymmetric substrate dimer row. Whether this difference is due to a differ- ence in attempt frequency or diffusion barrier could not be extracted from the experiments.

\section{ACKNOWLEDGMENTS}

This work is financially supported by the Stichting voor Fundamenteel Onderzoek der Materie (FOM, 03PR2208).
${ }^{1}$ B. S. Swartzentruber, Phys. Rev. Lett. 76, 459 (1996).

${ }^{2}$ B. Borovsky, M. Krueger, and E. Ganz, Phys. Rev. Lett. 78, 4229 (1997).

${ }^{3}$ H. J. W. Zandvliet, B. Poelsema, and B. S. Swartzentruber, Phys. Today 54, 40 (2001).

${ }^{4}$ Z. Zhang, F. Wu, H. J. W. Zandvliet, B. Poelsema, H. Metiu, and M. G. Lagally, Phys. Rev. Lett. 74, 3644 (1995).

${ }^{5}$ P. J. Bedrossian, Phys. Rev. Lett. 74, 3648 (1995).

${ }^{6}$ W. Wulfhekel, B. J. Hattink, H. J. W. Zandvliet, G. Rosenfeld, and B. Poelsema, Phys. Rev. Lett. 79, 2494 (1997).

${ }^{7}$ E. Zoethout, H. J. W. Zandvliet, W. Wulfhekel, G. Rosenfeld, and B. Poelsema, Phys. Rev. B 58, 16167 (1998).

${ }^{8}$ H. J. W. Zandvliet, T. M. Galea, E. Zoethout, and B. Poelsema, Phys. Rev. Lett. 84, 1523 (2000).

${ }^{9}$ T. M. Galea, C. Ordas, E. Zoethout, H. J. W. Zandvliet, and B.
Poelsema, Phys. Rev. B 62, 7206 (2000).

${ }^{10}$ H. J. W. Zandvliet, Phys. Rep. 388, 1 (2003).

${ }^{11}$ H. J. W. Zandvliet, Rev. Mod. Phys. 72, 593 (2000).

${ }^{12}$ R. M. Tromp, R. J. Hamers, and J. E. Demuth, Phys. Rev. Lett. 55, 1303 (1985).

${ }^{13}$ X. R. Qin and M. G. Lagally, Phys. Rev. B 59, 7293 (1999).

${ }^{14}$ K. Hata, S. Yasuda, and H. Shigekawa, Phys. Rev. B 60, 8164 (1999).

${ }^{15}$ D. Dijkkamp, E. J. van Loenen, and H. B. Elswijk, in Ordering at Surfaces and Interfaces, edited by A. Yoshimori, T. Shinjo, and H. Watanbe (Springer-Verlag, Berlin, 1992), p. 85.

${ }^{16}$ J. M. Carpinelli and B. S. Swartzentruber, Phys. Rev. B 58, R13423 (1998).

${ }^{17}$ H. J. W. Zandvliet, R. van Gastel, O. Gurlu, and B. Poelsema, Phys. Lett. A 326, 457 (2004). 